Article

\title{
Vertical Transmission of Extended-Spectrum, Beta-Lactamase-Producing Enterobacteriaceae during Preterm Delivery: A Prospective Study
}

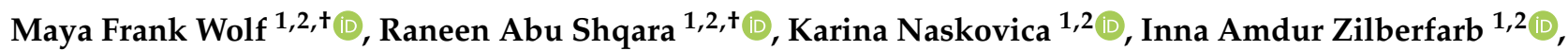

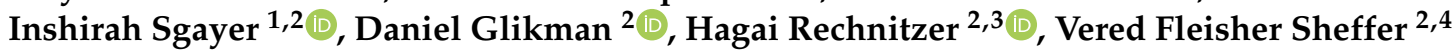 \\ and Jacob Bornstein 1,2,*(D) \\ 1 Department of Obstetrics and Gynecology, Galilee Medical Center, Nahariya 22100, Israel; \\ MayaW@gmc.gov.il (M.F.W.); rabushqara@gmail.com (R.A.S.); karina.naskovica@gmail.com (K.N.); \\ innazilberfarb@gmail.com (I.A.Z.); inshirah.sg.sh@gmail.com (I.S.) \\ 2 Azrieli Faculty of Medicine, Bar Ilan University, Safed 1311502, Israel; Daniel.glikman@biu.ac.il (D.G.); \\ hagair@gmc.gov.il (H.R.); VeredF@gmc.gov.il (V.F.S.) \\ 3 Clinical Microbiology Laboratory, Galilee Medical Center, Nahariya 22100, Israel \\ 4 Neonatal Intensive Care Unit, Galilee Medical Center, Nahariya 22100, Israel \\ * Correspondence: mdjacob@gmail.com; Tel.: +972-50-7887631 \\ + These authors contributed equally to this work.
}

Citation: Frank Wolf, M.; Abu Shqara, R.; Naskovica, K.; Zilberfarb, I.A.; Sgayer, I.; Glikman, D.; Rechnitzer, H.; Fleisher Sheffer, V.; Bornstein, J. Vertical Transmission of Extended-Spectrum,

Beta-Lactamase-Producing Enterobacteriaceae during Preterm Delivery: A Prospective Study. Microorganisms 2021, 9, 506. https:// doi.org/10.3390/microorganisms9030506

Academic Editor: Sander Smits

Received: 30 January 2021

Accepted: 24 February 2021

Published: 27 February 2021

Publisher's Note: MDPI stays neutral with regard to jurisdictional claims in published maps and institutional affiliations.

Copyright: (c) 2021 by the authors. Licensee MDPI, Basel, Switzerland. This article is an open access article distributed under the terms and conditions of the Creative Commons Attribution (CC BY) license (https:/ / creativecommons.org/licenses/by/ $4.0 /)$

\begin{abstract}
Maternal carriage and vertical transmission of extended-spectrum, beta-lactamase-producing Enterobacteriaceae (ESBL-E), such as Escherichia coli, hamper the treatment of infections, resulting in high morbidity. E. coli is the most frequent cause of early-onset neonatal sepsis (EOS) in preterm infants, where ESBL-E are more frequently isolated. In this prospective, case-controlled study, maternal rectovaginal ESBL-E colonization and vertical transmission to preterm infants were assessed in 160 women with preterm premature rupture of membranes (PPROM; 57.4\%) or preterm labor (42.6\%); additional cultures were obtained from the placenta, amnion, and umbilical cord during preterm labor. Maternal and neonatal ESBL-E-carriage rates were $17.5 \%$ and $12.9 \%$, respectively, and the vertical-transmission rate was 50\%. Maternal ESBL-E colonization among women with PPROM was $21.3 \%$, and in women with premature labor it was $12.6 \%$. No correlation was observed between maternal ESBL-E-colonization and previous hospitalization or antibiotic administration during pregnancy. However, a correlation was found between placental inflammation and maternal ESBL-E colonization $(p=0.007)$. ESBL-E-colonized infants were delivered at an earlier gestational age and were more likely to have complications. Thus, the high ESBL-E carriage rate in women with threatened preterm labor, without obvious risk factors for carriage, and a high vertical transmission rate, combined with a correlation between placental inflammation and ESBL-E carriage, support maternalneonatal ESBL-E-colonization surveillance and active measures to prevent ESBL-E-related EOS.
\end{abstract}

Keywords: extended-spectrum, beta-lactamase-producing Enterobacteriaceae; Escherichia coli; maternal colonization; preterm birth

\section{Introduction}

Global preterm birth rates have exhibited an increasing trend over the past two decades [1]. Preterm neonates who survive are at greater risk of a range of short-term and long-term morbidities [1-3]. Neonatal sepsis remains a major cause of morbidity and mortality during the neonatal period, despite significant advancements in perinatal care over the last few decades [4]. Group B Streptococcus (GBS) and Escherichia coli are the most common causes of all cases of neonatal early-onset sepsis (EOS) [5,6], while Klebsiella pneumoniae and E. coli are the most frequent causative organisms in most low- and middleincome countries $[7,8]$. In very preterm infants, the incidence of EOS is greater with E. coli than with GBS $[9,10]$. 
E. coli strains causing EOS exhibit a higher resistance to ampicillin, gentamicin, and thirdgeneration cephalosporins in preterm infants than in term infants $(93.3 \%$ vs. $48.4 \%, p<0.01)[8,11]$. Neonatal infections with extended-spectrum beta-lactamase (ESBL)-producing Enterobacteriaceae (ESBL-E), such as cefotaxime-resistant E. coli, are associated with higher mortality rates, longer neonatal intensive care unit (NICU) stays, and reduced clinical and microbiological responses [12]. Neonates could be exposed to ESBL-E during parturition (via passage through the birth canal) or before delivery (after rupturing of the amniotic membrane) [5,13]. Thus, the maternal rectovaginal ESBL-E-carriage rate has recently gained increasing attention. The reported prevalence of vaginal ESBL-E colonization during pregnancy ranges from $4.0 \%-19.9 \%[14,15]$, and varies with different geographical locations, e.g., it is as high as $15 \%-17 \%$ in Africa versus $7 \%$ in Argentina $[14,16]$. The vertical-transmission rate also varies; previous data revealed a high maternal-neonataltransmission rate of $35.7 \%$ in Norway [17], whereas other studies did not report a significant correlation between the maternal and neonatal ESBL-E status [18].

Although most cases of histological chorioamnionitis are sub-clinical in nature [19], infection may be implicated in a substantial proportion of women who undergo preterm labor [20-22]. The role of maternal rectovaginal ESBL-E carriage remains unknown [23], and it is unclear whether it is correlated with preterm birth.

Very-low-birthweight (VLBW) infants are at the greatest risk of developing invasive disease, because of their compromised immunity. These infants are more likely to develop sepsis from Gram-negative organisms, including E. coli, rather than GBS; therefore, the greatest risk of maternal ESBL-E colonization and possible vertical transmission is for VLBW infants $[5,8,9]$.

This study aimed to evaluate the rate of ESBL-E colonization among women in preterm labor and women with preterm premature rupture of membranes (PPROM), incidence of maternal vertical transmission, intrauterine inflammation, and the clinical significance of ESBL-E in preterm infants. We assumed that ESBL-E-colonized infants would be delivered at an earlier gestational age (GA) and develop more prematurity-related complications. The principal conclusions of this study are that women with threatened premature delivery had relatively high rates of ESBL-E rectovaginal colonization (17.5\%) and vertical transmission $(50 \%)$.

\section{Materials and Methods}

\subsection{Study Description}

This prospective, case-controlled study was carried out in the delivery rooms and maternal-fetal unit of a single, tertiary, university-affiliated hospital from March 2017 to December 2019. The Institutional Review Board of Galilee Medical Center approved the study (approval code 0188-16 NHR). The ClinicalTrials.gov identifier is NCT03251885. The study included women presenting with threatened preterm delivery at $<37$ weeks. Preterm labor was diagnosed as uterine contractions and cervical dilatation over time or PPROM. PPROM diagnosis was made based on a history of fluid leakage accompanied by pooling of amniotic fluid observed upon sterile speculum examination. If there was no clear pooling, the AmniSure ROM test (AmniSure International LLC, Boston, MA, USA), an immune-chromatography method, was used to confirm the diagnosis. Exclusion criteria were active preterm labor with cervical dilatation $>6 \mathrm{~cm}$, cases where an emergency cesarean section was performed owing to fetal distress, bleeding from placenta previa, acute placental abruption, and cord prolapse. Written informed consent was obtained from all participants. This study was performed in accordance with the code of ethics of the World Medical Association (Declaration of Helsinki) for experiments involving humans.

All women included in this study were hospitalized for conservative treatment, including the use of tocolysis, magnesium sulfate administration for neuroprotection before 32 weeks of gestation (if indicated), antenatal corticosteroids, and prophylactic antibiotic courses in cases of PPROM. 


\subsection{Maternal Screening}

A routine maternal rectovaginal swab for GBS was obtained in cases of threatened premature labor or PPROM. In addition, another rectovaginal swab for ESBL-E was performed upon admission, according to the study protocol. A positive ESBL-E colonization result, as a single finding, was not considered an indication for antibiotic treatment. Furthermore, during preterm labor, surface swab cultures were routinely obtained from the placenta, amnion, and umbilical cord to detect colonization and infection.

\subsection{Neonatal Screening and Birth Cultures}

Infants who were admitted to the NICU were screened for ESBL-E and included in this study. Rectal samples were obtained upon admission to the NICU to detect early colonization and implement appropriate neonatal isolation measures [17,24]. Maternal, neonatal, and placental cultures were immediately placed in Amies transport nutrient medium (COPAN, Brescia BS, Italy) and transferred to the microbiology laboratory. The results were reported within $48-72 \mathrm{~h}$.

\subsection{Identification of ESBL Bacteria in Culture and Susceptibility Tests}

All specimens were cultured on CHROMagar ESBL (HY-Labs, Rehovot, Israel) growth medium and incubated for $18-24 \mathrm{~h}$ at $35^{\circ} \mathrm{C}$. This growth medium enables selective growth of mainly ESBL bacteria, and each bacterial species is characterized by a different colony color [24]. Final bacterial identification was made by VITEK MS systems (bioMérieux SA, Marcy-l'Étoile, France). Antibiotic susceptibility tests and final identification of the mechanism of ESBL resistance was performed by VITEK 2 systems (bioMérieux SA). The VITEK 2 expert system allows ESBL phenotype identification of E. coli, Klebsiella spp., and Proteus spp. This research focused on E. coli and Klebsiella spp. as they are the two most common ESBL-E. Susceptibility patterns to non-beta-lactam antimicrobials were compared in cases where ESBL-E were found in mothers and neonates.

\subsection{Risk Factors for Maternal ESBL Carriage}

We retrieved detailed patient information in terms of maternal demographics, obstetric histories, and antepartum records. Data on the hospitalization course were also collected, namely the GBS rectovaginal carriage status, urinary culture test results, administration of antibiotics during the current pregnancy, and previous hospitalizations during the current pregnancy.

\subsection{Risk Factors for Vertical Transmission of ESBL}

Data regarding antibiotic treatment during the week before delivery, PPROM, the latency period duration (defined as the period from PPROM until delivery), the mode of delivery, intrapartum fever, intrapartum antibiotic administration as indicated, birth weight (g), and the GA at delivery were obtained. After each preterm delivery, the placenta was subjected to histopathological examination for signs of inflammation showing neutrophilic infiltrate of the membrane, chorion, or subchorionic plate fibrin, scored by a specialized pathologist, as follows: grade 1 , subchorionitis; grade 2 , chorionitis; or grade 3 , chorioamnionitis.

\subsection{Neonatal Outcome}

The appearance, pulse, grimace, activity, and respiration (APGAR) score; cord $\mathrm{pH}$; antibiotic administration; NICU admission; and complications were recorded. The major complications included the following: (a) respiratory distress syndrome (RDS), defined as progressive respiratory failure manifested by an increase in dyspnea and oxygen requirement in conjunction with a characteristic radiologic pattern (including atelectasis with aerated airways and pulmonary edema); (b) necrotizing enterocolitis (NEC), defined by abdominal distention, bilious vomiting or gastric aspirate, and rectal bleeding, along with intestinal intramural gas on abdominal X-ray; (c) intraventricular hemorrhage (IVH), diagnosed by clinical features of irritability, apnea, or seizures, and confirmed by cranial ultra- 
sound or magnetic resonance imaging (MRI); and (d) bronchopulmonary dysplasia (BPD), a condition of chronic lung disease due to the disruption of pulmonary development, typically diagnosed when the neonate shows respiratory distress after 28 days of age or past 36 weeks post-conceptional age. The rate of ventilation support was also recorded, defined by the need for either noninvasive ventilation, such as Neopuff T-piece resuscitator and continuous positive airway pressure (CPAP), or invasive mechanical ventilation. Finally, neonatal sepsis and death rate were recorded. Neonatal outcomes were compared between the NICU infants with or without ESBL-E colonization.

\subsection{Statistical Analysis}

Continuous variables are presented as the mean \pm standard deviation (SD), or as median and range values. Qualitative variables are presented as frequencies and percentages. Continuous variables were compared between the two groups using either the independent sample $t$-test or the Mann-Whitney test, based on the sample sizes of the groups and the distribution shapes of the variables. Categorical variables were analyzed using Pearson's chi-squared test or Fisher's exact test. To examine whether neonatal ESBL-E status was correlated with complications, a multivariate logistic regression for neonatal outcomes was performed and adjusted to GA, neonatal gender, and mode of delivery, and was based on neonatal colonization by a backward elimination regression. A multivariate linear regression model was used for NICU hospitalization length, adjusted to the abovementioned variables. A two-tailed $p$ value of $<0.05$ was considered significantly different. To detect neonatal morbidity, pooled ratios for RDS, NEC, IVH, and BPD were determined. Furthermore, pooled ratios for positive cultures from amniotic fluid, placentas, and umbilical cords were determined. Statistical analysis was performed by the Statistics Unit at Galilee Medical Center using IBM SPSS Statistics for Windows, version 25.0 (IBM Corp., Armonk, NY, USA).

\section{Results}

\subsection{Characteristics of the Study Population}

In total, 160 pregnant women with threatened premature labor were recruited for the study (Figure 1); $42.6 \%$ of these patients had premature labor with uterine contractions and cervical dynamic change, whereas $57.4 \%$ had PPROM. No significant differences in the background data were observed between the two groups, except for previous preterm birth (Table 1). GA at recruitment was similar between the two groups. Of the 160 patients, 139 were screened for rectovaginal GBS at the time of admission; carriage rate was similar between both groups (Table 2).

Table 1. Baseline characteristics of women participating in the study.

\begin{tabular}{cccccc}
\hline $\begin{array}{c}\text { Baseline } \\
\text { Characteristic }\end{array}$ & $\begin{array}{c}\text { Cohort } \\
(\boldsymbol{n}=\mathbf{1 6 0})\end{array}$ & $\begin{array}{c}\text { Preterm Labor } \\
(\boldsymbol{n}=\mathbf{7 1})\end{array}$ & $\begin{array}{c}\text { PPROM } \\
(\boldsymbol{n}=\mathbf{8 9})\end{array}$ & $\boldsymbol{p}$-Value \\
\hline Maternal Age (y) & Mean \pm SD & $29.6 \pm 6.3$ & $28.8 \pm 6.2$ & $29.9 \pm 6.4$ & 0.29 \\
Parity & Median (range) & $2(1-10)$ & $2(1-5)$ & $2(1-10)$ & 0.295 \\
Gravidity & Median (range) & $2(1-14)$ & $2(1-7)$ & $2(1-14)$ & 0.191 \\
Previous Preterm Birth & & $36(22.5 \%)$ & $21(29.6 \%)$ & $15(17 \%)$ & 0.033 \\
Cervical Cerclage in & $11(6.8 \%)$ & $3(4.2 \%)$ & $8(8.9 \%)$ & 0.358 \\
Situ & $16(10.0 \%)$ & $9(12.7 \%)$ & $7(7.9 \%)$ & 0.284 \\
Maternal Diabetes & $7(4.5 \%)$ & $5(7.0 \%)$ & $2(2.2 \%)$ & 0.132 \\
Multiple Gestations & & &
\end{tabular}

PPROM: preterm premature rupture of membranes; SD: standard deviation. 
Table 2. Obstetric outcomes stratified by PPROM and preterm labor.

\begin{tabular}{|c|c|c|c|c|c|}
\hline Variable & & $\begin{array}{l}\text { Cohort } \\
(n=160)\end{array}$ & $\begin{array}{l}\text { Preterm Labor } \\
\quad(n=71)\end{array}$ & $\begin{array}{c}\text { PPROM } \\
(n=89)\end{array}$ & $p$-Value \\
\hline ESBL-E Status & & $28(\% 5.17)$ & $9(12.6 \%)$ & $19(21.3 \%)$ & 0.292 \\
\hline Sampling Week & Median (range) & $33.64(24.1-36.9)$ & $33.57(24.9-36.9)$ & $33.71(24.1-36.9)$ & 0.481 \\
\hline GBS Carriage Status & $n=139$ & $22 / 139(15.8 \%)$ & $11 / 59(18.6 \%)$ & $11 / 80(13.8 \%)$ & 0.485 \\
\hline Delivery Week & Median (range) & $35.8(25.4-42.3)$ & $37.0(29.1-40.6)$ & $34.6(25.4-42.3)$ & $<0.001$ \\
\hline $\begin{array}{l}\text { Delivery Mode among } \\
\text { Final Sample }\end{array}$ & Cesarean & $45(28.1 \%)$ & $13(18.3 \%)$ & $32(36.0 \%)$ & 0.032 \\
\hline
\end{tabular}

ESBL-E: extended-spectrum, beta-lactamase-producing Enterobacteriaceae; GBS: group B Streptococcus; PPROM: preterm premature rupture of membranes.

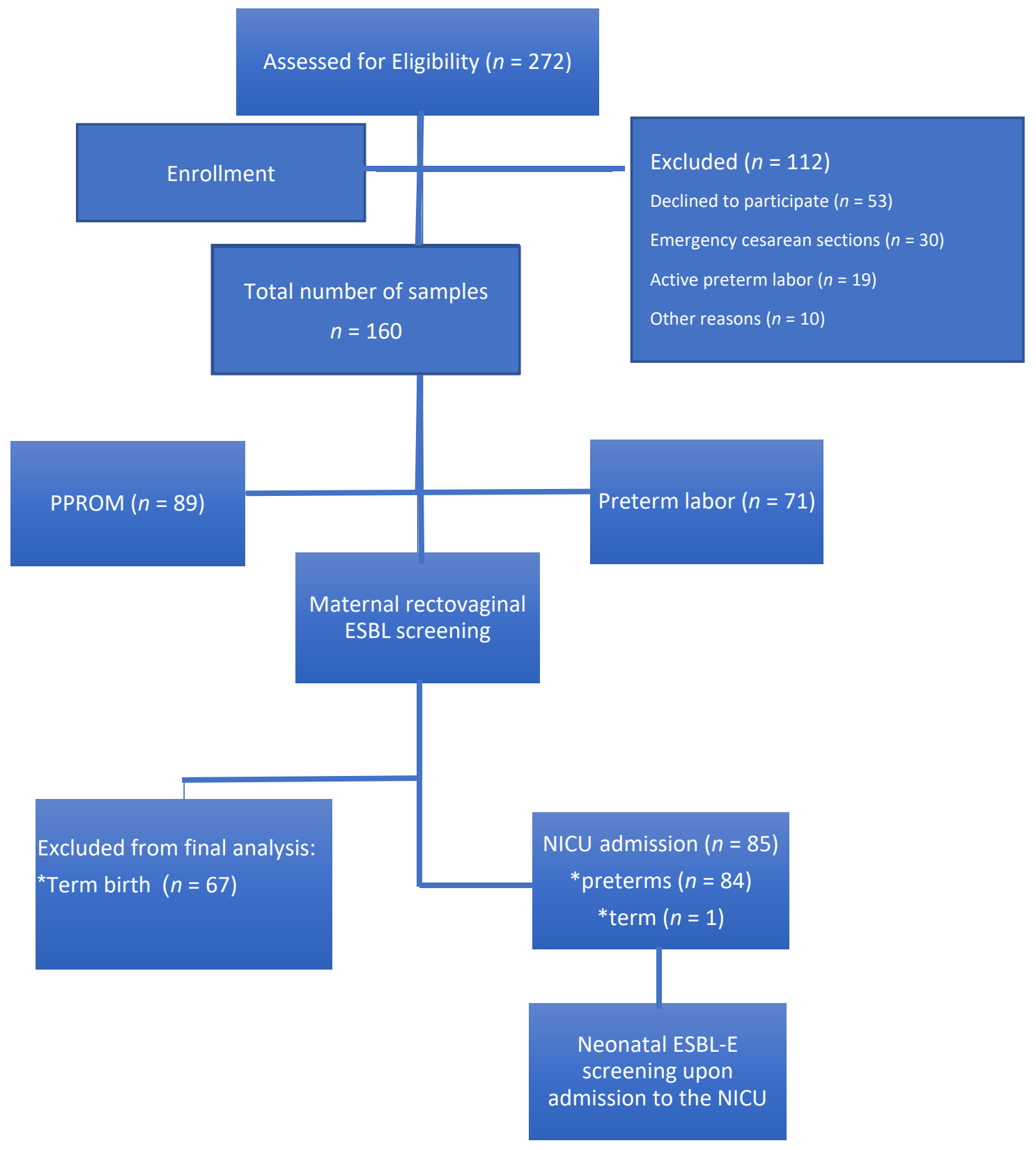

Figure 1. Study population. ESBL-E: extended-spectrum beta-lactamase-producing Enterobacteriaceae; NICU: neonatal intensive care unit; PPROM: preterm premature rupture of membranes. 


\subsection{Prevalence of Maternal ESBL-E Colonization and Risk Factors}

The prevalence of maternal ESBL colonization was $17.5 \%(n=28)$ among all patients, $21.3 \%(n=19)$ in women with PPROM, and $12.6 \%(n=9)$ in women with premature labor $(p=0.292 ;$ Table 2). ESBL E. coli was isolated from most women with ESBL colonization $(85.7 \%, n=24 / 28)$, followed by ESBL K. pneumoniae (14.3\%, $n=4 / 28$; Figure 2). No significant correlations were observed between the maternal ESBL-E-colonization status and maternal baseline characteristics, including age, parity, gravidity, or previous hospitalization. In addition, no correlation was observed between the maternal ESBL-E-colonization status and the GBS-carriage status, administration of antibiotics, or urinary tract infections during pregnancy (Table 3).

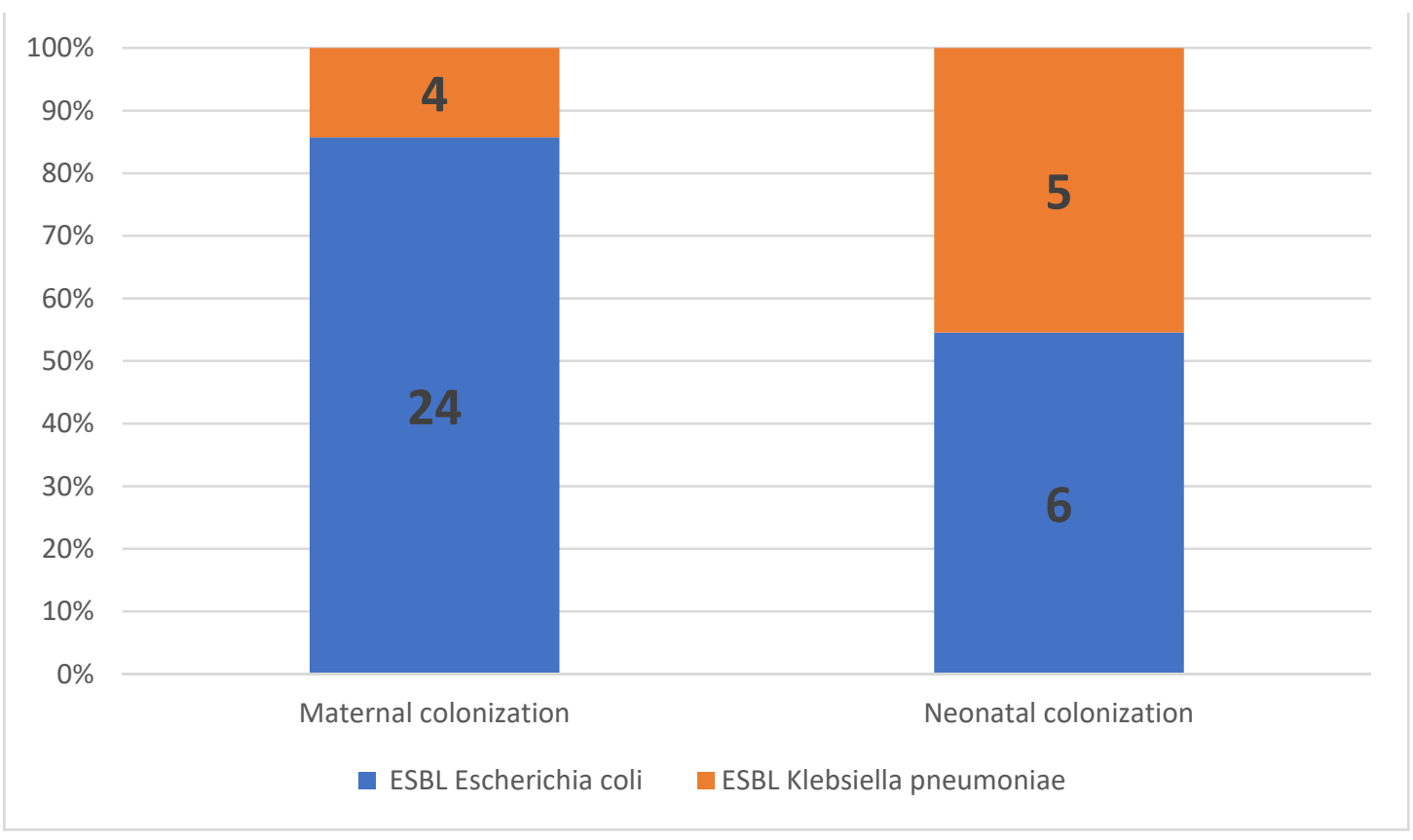

Figure 2. Proportion of extended-spectrum, beta-lactamase-producing Enterobacteriaceae (ESBL-E) isolates in maternal and neonatal colonization.

Table 3. Association between maternal characteristics and ESBL-E colonization status.

\begin{tabular}{|c|c|c|c|c|c|}
\hline Variable & & Cohort $(n=160)$ & $\begin{array}{c}\text { Negative Maternal } \\
\text { ESBL-E-Colonization }(n=132)\end{array}$ & $\begin{array}{l}\text { Positive Maternal ESBL-E } \\
\text { Colonization }(n=28)\end{array}$ & $p$-Value \\
\hline Sampling Week & Mean \pm SD & $32.63 \pm 3.45$ & $32.75 \pm 3.42$ & $32.09 \pm 3.61$ & 0.356 \\
\hline Maternal Age (years) & Mean \pm SD & $29.61 \pm 6.36$ & $29.43 \pm 6.02$ & $30.54 \pm 7.93$ & 0.251 \\
\hline Gravidity & Mean \pm SD & $2.55 \pm 1.86$ & $2.48 \pm 1.65$ & $2.88 \pm 2.70$ & 0.301 \\
\hline Parity & Mean \pm SD & $1.95 \pm 1.29$ & $1.88 \pm 1.08$ & $2.31 \pm 2.015$ & 0.253 \\
\hline Hospital Admissions & Median(range) & $0.54 \pm 0.78$ & $0.56 \pm 0.79$ & $0.46 \pm 0.71$ & 0.331 \\
\hline Positive GBS Status & $n=139$ & $22 / 139(15.8 \%)$ & $19 / 117(16.2 \%)$ & $3 / 22(13.6 \%)$ & 1.00 \\
\hline UTI in Current Pregnancy & & $19(11.8 \%)$ & $14(10.6 \%)$ & $5(17.9 \%)$ & 0.332 \\
\hline $\begin{array}{l}\text { Antibiotic Administration } \\
\text { (Prior to Sampling) }\end{array}$ & & $12(7.5 \%)$ & $8(6)$ & $4(14.2 \%)$ & 0.110 \\
\hline
\end{tabular}

ESBL-E: extended-spectrum, beta-lactamase-producing Enterobacteriaceae; GBS: group B Streptococcus; UTI: urinary tract infection.

\subsection{Maternal ESBL-E Colonization and Obstetric Outcomes}

In total, 160 women were included in the outcome analysis. The median GA at delivery was 35.8 (25.4-42.3) weeks, and the cesarean section rate was $29.6 \%$. No correlation was observed between maternal ESBL-E colonization status and GA at delivery (Table 4). Eighty ESBL-E preterm birth cultures (placenta, umbilical cord, and amnion) were analyzed, and no significant differences were observed in the positive ESBL-E birth cultures between ESBL-E-colonized and non-colonized mothers $(p=0.062)$. However, in 60 placenta samples 
Table 4).

that were submitted for histopathological examination, a correlation was observed between placental inflammation signs and maternal ESBL-E colonization status $(p=0.007$;

Table 4. Association between maternal ESBL-E colonization and perinatal outcomes.

\begin{tabular}{|c|c|c|c|c|c|}
\hline Variable & & $\begin{array}{l}\text { Cohort } \\
(n=160)\end{array}$ & $\begin{array}{l}\text { Negative Maternal } \\
\text { ESBL-E-Colonization } \\
\quad(n=132)\end{array}$ & $\begin{array}{l}\text { Positive Maternal ESBL-E } \\
\text { Colonization } \\
(n=28)\end{array}$ & $p$-Value \\
\hline Delivery Week & Median (range) & $35.8(25.4-42.3)$ & $35.9(26.6-42.3)$ & $35.4(25.4-40.0)$ & 0.659 \\
\hline Delivery Mode & Cesarean & $45(28.1 \%)$ & $36(27.3 \%)$ & $9(32.1 \%)$ & 0.60 \\
\hline $\begin{array}{c}\text { Placental Inflammation } \\
\text { Signs }\end{array}$ & $n=61$ & $17 / 61(27.9 \%)$ & $10 / 50(20 \%)$ & $7 / 11(63.6 \%)$ & 0.007 \\
\hline $\begin{array}{l}\text { ESBL-E Isolates (Placenta, } \\
\text { Cord, Amnion) }\end{array}$ & $\begin{array}{l}\text { Pooled ratio } \\
\qquad(n=80)\end{array}$ & $5 / 80(6.3 \%)$ & $2 / 63(3.2 \%)$ & $3 / 17(17.6 \%)$ & 0.062 \\
\hline \multicolumn{6}{|l|}{ Perinatal Outcome } \\
\hline NICU Admission & $n=85$ & $85(53.1 \%)$ & $69 / 132(52.2 \%)$ & $16 / 28(57.1 \%)$ & 0.835 \\
\hline $\begin{array}{l}\text { Positive Neonatal } \\
\text { ESBL-E-Colonization } \\
\text { (at Admission) }\end{array}$ & $n=85$ & $11 / 85(12.9 \%)$ & $3 / 69(4.3 \%)$ & $8 / 16(50 \%)$ & $<0.001$ \\
\hline
\end{tabular}

ESBL-E: extended-spectrum, beta-lactamase-producing Enterobacteriaceae; NICU: neonatal intensive care unit; SD: standard deviation.

\subsection{Neonatal Outcomes}

Eighty-five neonates (53.1\%) were admitted to the NICU; among them, 84 were preterms $(\mathrm{GA}<37)$ and one was delivered at term $(\mathrm{GA}=39$, admitted due to fever at birth, negative for ESBL-E). Of the 85, 18.9\% were born to ESBL-E-colonized mothers. The overall ESBL-E-colonization rate among preterm infants was $13 \%(11 / 84)$. ESBL E. coli was isolated from 6 out of 11 preterm infants (55\%), and ESBL K. pneumoniae was isolated from the remaining five preterm infants $(45 \%)$. Of note, ESBL K. pneumoniae colonization was more prevalent in the preterm infants than in the mothers (Figure 2).

ESBL-E-colonized neonates were born at an earlier GA (median $=30.3$ (25.4-34.0) vs. 34 (26.6-36.9) weeks, $p=0.006)$ and had a relatively lower birthweight $(1502.4 \pm 498.9 \mathrm{~g}$ vs. $2032.5 \pm 692.6 \mathrm{~g}, p=0.009)$ than neonates without ESBL-E colonization. No correlations were observed between the mode of delivery, antepartum or intrapartum antibiotic administration, latency period, presence of PPROM, intrapartum fever, and risk of neonatal ESBL-E colonization at the time of NICU admission (Table 5). Also, no correlation was observed between ESBL-E status in NICU-admitted neonates and the 5 min APGAR score $(<7)$ or cord $\mathrm{pH}(<7.10)$ (Tables 4 and 5$)$.

Univariate analysis revealed a correlation between hospitalization duration, antibiotic administration in the NICU, and neonatal complications with neonatal ESBL-E colonization status ( $p=0.049, p=0.03$, and $p=0.023$, respectively); no difference was observed in the need for ventilation support or perinatal mortality rate with respect to neonatal ESBLE status (Table 5). Multivariate analysis, used to evaluate the correlation between neonatal ESBL-E status and neonatal outcomes, adjusted for delivery week, gender, and mode of delivery, revealed that delivery week was uniformly correlated with all outcomes. Cesarean sections increased NICU hospitalization length (Table 6). Of note, one case of neonatal death occurred following ESBL-E vertical transmission in a preterm born at 28.2 weeks of gestation, owing to PPROM. The neonate received empiric antibiotic treatment with gentamicin and ampicillin, but presented with ESBL-E sepsis and subsequent peritonitis. Although the antibiotic was switched to meropenem, the infant did not survive. 
Table 5. Risk factors and outcomes of ESBL-E colonization among neonates in the NICU.

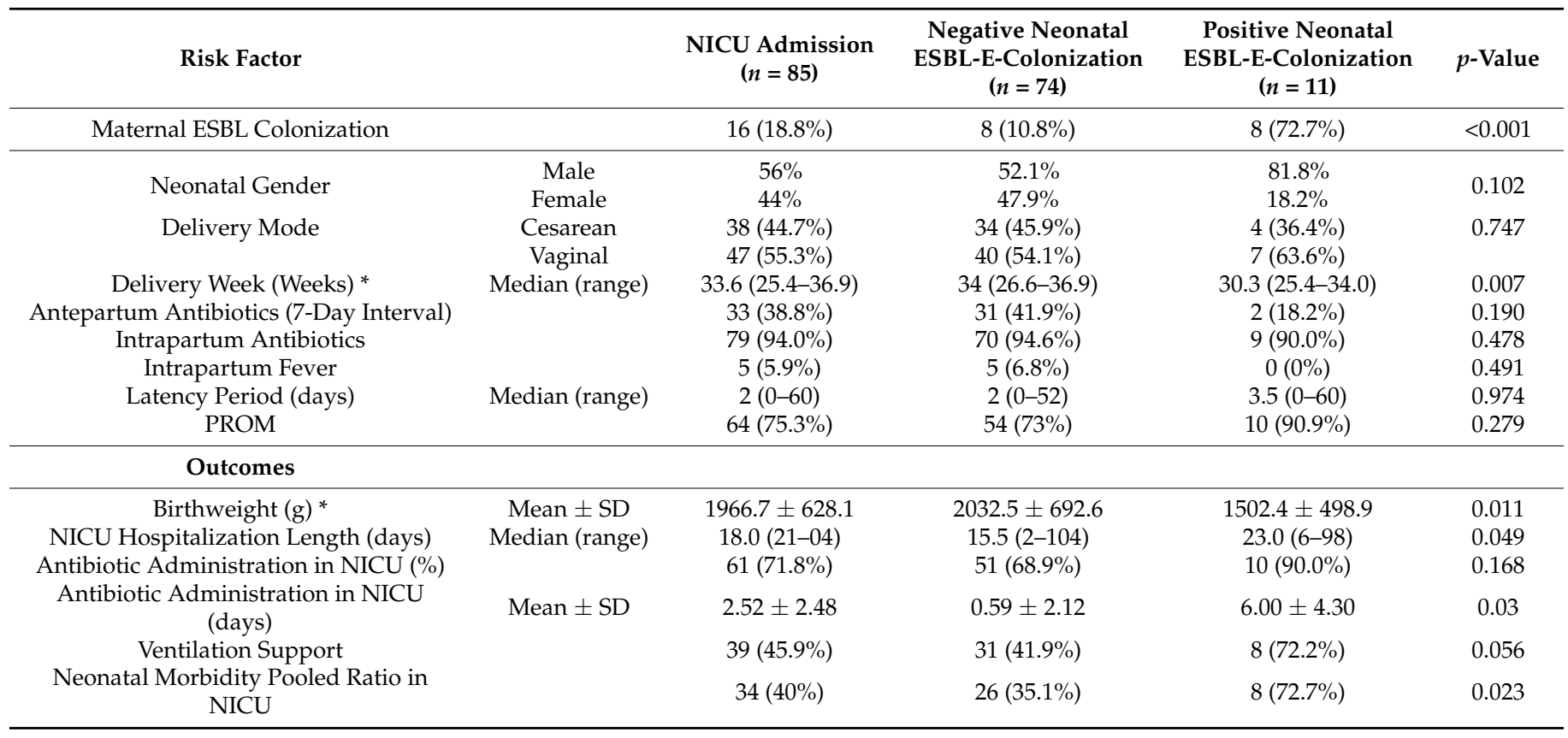

ESBL-E: extended-spectrum, beta-lactamase-producing Enterobacteriaceae; NICU: neonatal intensive care unit; PPROM: preterm premature rupture of membranes; SD: standard deviation. * Term neonate $(1 / 85)$ was excluded from the analysis of delivery week and birthweight.

Table 6. Multivariate regression: correlation between variables and neonatal outcome in NICU.

\begin{tabular}{|c|c|c|c|c|}
\hline $\begin{array}{c}\text { Neonatal } \\
\text { Complications } \\
\left(R^{2}=46.3 \%\right)\end{array}$ & $p$-Value & Odds Ratio & $\begin{array}{c}\text { 95\% CI } \\
\text { Lower Limit }\end{array}$ & $\begin{array}{c}95 \% \text { CI } \\
\text { Upper Limit }\end{array}$ \\
\hline Positive Neonatal ESBL-E-Colonization & 0.124 & 3.656 & 0.7000 & 18.984 \\
\hline Delivery Week & $p<0.001$ & 0.0170 & 0.002 & 0.164 \\
\hline Delivery Mode (Cesarean vs. Vaginal) & 0.647 & 1.302 & 0.420 & 4.034 \\
\hline Gender (Female vs. Male) & 0.076 & 0.322 & 0.092 & 1.125 \\
\hline $\begin{array}{l}\text { Need for Ventilation } \\
\qquad\left(R^{2}=58.0 \%\right)\end{array}$ & $p$-Value & Odds Ratio & $\begin{array}{c}\text { 95\% CI } \\
\text { Lower limit }\end{array}$ & $\begin{array}{l}95 \% \text { CI } \\
\text { Upper }\end{array}$ \\
\hline $\begin{array}{l}\text { Positive Neonatal ESBL-E-Colonization } \\
\text { (Positive versus Negative) }\end{array}$ & & & 0.1990 & 9.082 \\
\hline Delivery Mode (Cesarean versus Vaginal) & 0.776 & 1.208 & 0.329 & 4.432 \\
\hline Delivery Week & $p<0.001$ & 0.450 & 0.313 & 0.646 \\
\hline Gender (Female versus Male) & 0.363 & 0.559 & 0.160 & 1.955 \\
\hline $\begin{array}{l}\text { NICU Hospitalization Length } \\
\qquad\left(R^{2}=60.9 \%\right)\end{array}$ & $p$-Value & $\begin{array}{l}\text { B-Standardized } \\
\text { coefficients }\end{array}$ & & \\
\hline Positive Neonatal ESBL-E-Colonization & 0.179 & 0.097 & & \\
\hline Delivery Mode (Cesarean versus Vaginal) & $p<0.001$ & 0.291 & & \\
\hline Delivery Week & $p<0.001$ & -0.648 & & \\
\hline Gender (Female versus Male) & 0.190 & -0.093 & & \\
\hline
\end{tabular}

ESBL-E: extended-spectrum, beta-lactamase-producing Enterobacteriaceae; NICU: neonatal intensive care unit.

\subsection{Vertical Transmission of ESBL}

The maternal-neonatal transmission rate was $50 \%(8 / 16, p<0.001$; Figure 3$)$. Furthermore, $72.2 \%$ of ESBL-colonized preterm infants were born to an ESBL-colonized mother, whereas only $10 \%$ of ESBL-negative neonates were born to ESBL-colonized mothers $(p<0.001)$. Thus, maternal ESBL colonization is correlated with neonatal positive colonization status. We also observed identical non-beta-lactam antimicrobial suscepti- 
bility (to gentamicin, amikacin, trimethoprim/sulfa, and ciprofloxacin) in cases of paired maternal-neonatal ESBL-E colonization.

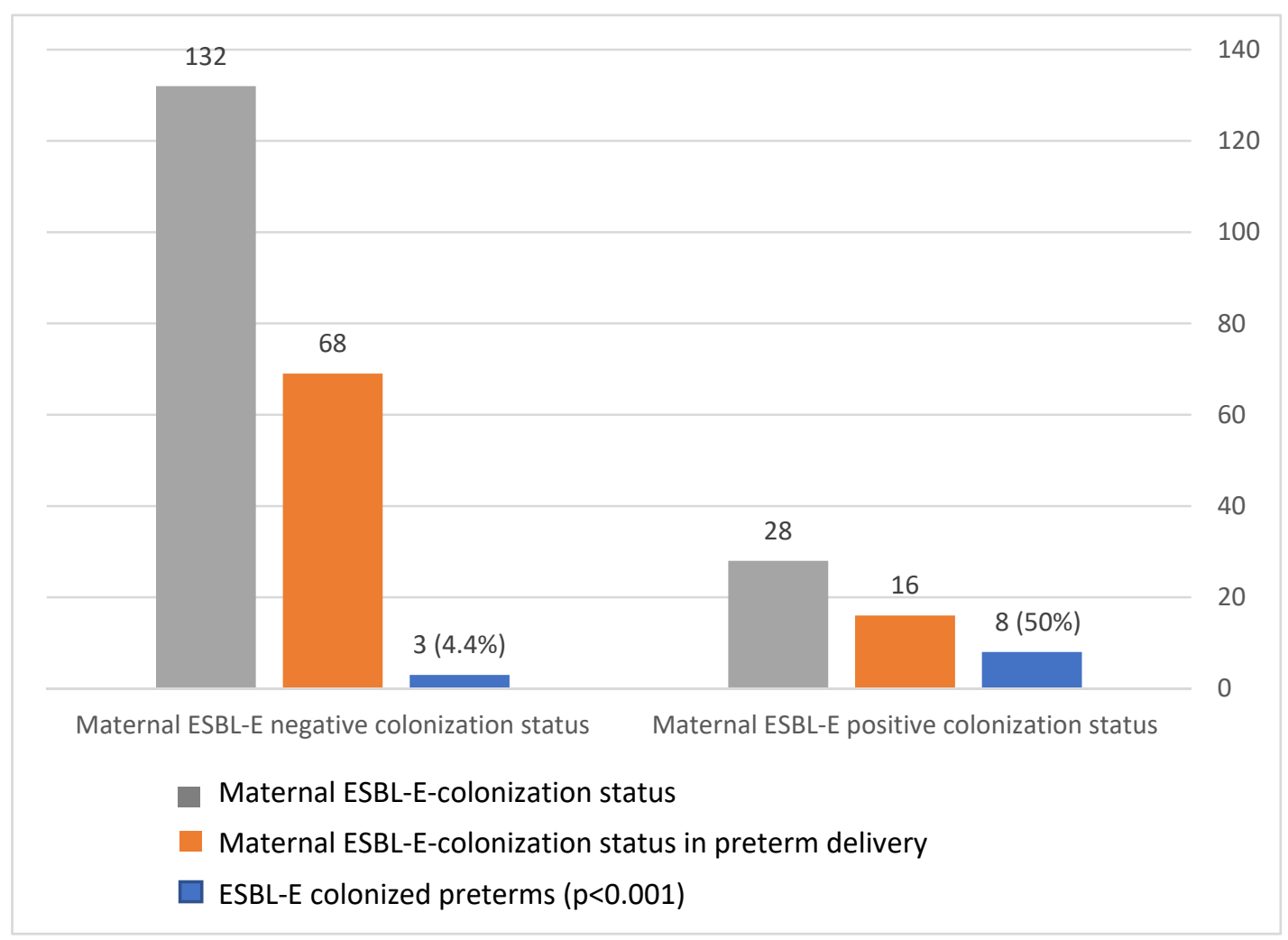

Figure 3. Extended-spectrum, beta-lactamase-producing Enterobacteriaceae (ESBL-E) transmission rate during preterm delivery.

\section{Discussion}

\subsection{Main Findings}

The primary findings of this study are the ESBL-E rectovaginal colonization rates in women with threatened premature delivery $(17.5 \%)$ and the high vertical-transmission rates $(50 \%)$. The ESBL-E-carriage rate of our study population was comparable to that reported in Africa $[14,16]$ and substantially higher than that observed in high- and middleincome countries, e.g., Norway (2.9\%) [17] and Argentina (5.4\%) [15], respectively. We also observed a slightly higher maternal-neonatal transmission rate than previously reported $(50 \%$ vs. $35 \%)[17,25]$. These findings suggest that maternal ESBL-E colonization constitutes a substantial risk for infant colonization.

Although a positive correlation was previously observed between maternal ESBLE colonization and urinary tract infections during pregnancy, antibiotic administration, and hospitalization [26], the present results do not support these findings, and no correlation between maternal ESBL-E-colonization status and urinary tract infection during pregnancy was observed. Nevertheless, our findings of a high ESBL-E carriage rate in women with threatened preterm labor, without obvious risk factors for carriage, and a high vertical transmission rate support maternal-neonatal ESBL-E-colonization surveillance in threatened preterm labor.

\subsection{Ascending Infection and Placental Involvement}

We observed a significant correlation between maternal ESBL-E colonization status and signs of histopathologic placental inflammation; however, no significant correlation was observed between maternal ESBL-E colonization and the pooled ratio of ESBL-E isolates from placenta, umbilical cord, and amnion cultures. These findings suggest that maternal ESBL-E colonization predisposes individuals to ascending infection and placental 
involvement. Ascending infection has been implicated as a cause for preterm delivery, as it promotes multiple physiological events, including increased levels of pro-inflammatory cytokines, membrane rupture, cervical ripening, and uterine contraction [27]. In this study, ESBL-E-colonized preterm infants were more likely to be born at an earlier GA. Of note, neonatal screening was conducted on the first day of NICU admission; hence, it appears that the preterm neonates acquired ESBL-E through the birth canal or more likely predelivery [28], resulting in a sub-clinical infection that may have initiated the preterm birth. Previous data support the vertical-transmission theory by showing that mother-neonate pairs are colonized with identical ESBL-E, based on species identification and antibiograms with $70 \%$ shared molecular-fingerprinting patterns $[25,29,30]$.

\subsection{Clinical Outcomes}

The correlation between neonatal ESBL-E colonization and RDS or the need for ventilation support has been previously reported [31]. Multivariate analysis revealed that the primary factor affecting newborn complications was GA at birth; we did not observe a significant association between neonatal ESBL-E status and neonatal complications and hospitalization length in the NICU, probably because of the small sample size. With adjustment for confounders, we did not observe a correlation between neonatal ESBL-E status and the need for ventilation or complications. Previous studies have indicated a high fatality rate in neonatal ESBL-E infections, which was most likely due to inadequate antibiotic treatment [28]. Although we did not observe a higher fatality rate in ESBL-E-colonized preterm infants, the one case of neonatal mortality occurred in an ESBL-E-colonized newborn with ESBL-E sepsis.

\subsection{Strengths and Limitations}

There are some limitations associated with the study. Specifically, we employed a relatively small sample size, and only NICU-admitted infants were screened for ESBL-E. Therefore, term infants that were not admitted to the NICU but had potential ESBL-E colonization were excluded from the final analysis. Another limitation is the inability to prove direct vertical transmission, since molecular typing of the ESBL-E-positive isolates was not performed, although we observed identical antimicrobial susceptibility patterns in cases of maternal-neonatal ESBL-E colonization. In addition, Mycoplasma and Ureaplasma colonization rates are not reported in this study, since these species are not routinely cultured at the bacteriology laboratory of our institute. The coexistence of ESBL-E and Mycoplasma could be a possible confounder in the interpretation of the correlation between ESBL-E and placental inflammation. Despite these limitations, our study has several unique strengths, in that it was a prospective study with controlled groups of ESBL-Ecolonized and non-colonized mothers and preterm infants. This study allowed us to compare risk factors and outcomes. To the best of our knowledge, this is the first study to analyze potential correlations between maternal ESBL-E-colonization status and cultures obtained during delivery from the placenta, amnion, and umbilical cord.

\subsection{Implications}

Antenatal screening for GBS carriage is a routine part of antenatal care in most developed countries, including the United States [32]. The prevalence of rectovaginal GBS colonization in pregnant women ranges between $10 \%$ and $30 \%$ [33,34]. Although E. coli (including ESBL E. coli) is currently the most common pathogen in preterm infants, and is associated with a higher mortality rate than that of GBS $[14,16,25,34]$, no universal screening test has been recommended for detecting ESBL-E carriage. In addition, although intrapartum GBS-targeting prophylactic antibiotics are usually administered in preterm deliveries in countries where routine screening for GBS is not performed, these antibiotics do not exhibit activity against ESBL-E [32,35]. This factor may emphasize the need for universal surveillance for ESBL-E among women with threatened premature labor. 
Moreover, the relatively high prevalence of ESBL-E colonization among women with threatened premature delivery, no obvious risk factors for colonization, and high ESBLE-transmission rate observed in this study is worrisome. The greatest risk of maternal ESBL-E colonization and possible vertical transmission is for VLBW infants, since these infants are more likely to develop sepsis from Gram-negative organisms $[5,8,9]$.

The correlation between placental inflammation and ESBL-E carriage and the identical non-beta-lactam antimicrobial susceptibility observed in paired maternal-neonatal ESBL-E colonization supports maternal-neonatal ESBL-E-colonization surveillance and active measures to prevent ESBL-E-related EOS. Additional studies are required to further explore the effect of maternal ESBL-E colonization on short- and long-term neonatal health outcomes, and to evaluate the efficacies of various intrapartum antibiotic regimens in ESBL-E-colonized mothers.

Author Contributions: Conceptualization, M.F.W. and J.B.; data curation, K.N., R.A.S., I.A.Z., I.S., H.R., and V.F.S.; formal analysis, M.F.W., D.G., H.R., V.F.S., and J.B.; investigation, I.S.; methodology, M.F.W. and D.G.; project administration, M.F.W.; validation, J.B.; writing-original draft, M.F.W., R.A.S., and J.B.; and writing—review and editing, M.F.W., K.N., R.A.S., I.A.Z., I.S., D.G, H.R., V.F.S., and J.B. All authors have read and agreed to the published version of the manuscript.

Funding: This research received no external funding.

Institutional Review Board Statement: The study was conducted according to the guidelines of the Declaration of Helsinki and approved by the Institutional Review Board of Galilee Medical Center (approval code 0188-16 NHR, date of approval: 13 March 2017). The ClinicalTrials.gov identifier is NCT03251885.

Informed Consent Statement: Written informed consent was obtained from all participants.

Data Availability Statement: Not applicable.

Acknowledgments: Not applicable.

Conflicts of Interest: The authors declare no conflict of interest.

$\begin{array}{ll}\text { Abbreviations } & \\ \text { ESBL-E } & \text { extended-spectrum beta-lactamase producing Enterobacteriaceae. } \\ \text { EOS early } & \text { onset neonatal sepsis } \\ \text { PPROM } & \text { preterm premature rupture of membranes } \\ \text { ESBL } & \text { extended-spectrum beta-lactamase } \\ \text { GBS } & \text { Group B Streptococcus } \\ \text { E. coli } & \text { Escherichia coli } \\ \text { NICU } & \text { neonatal intensive care unit } \\ \text { VLBW } & \text { very-low-birthweight } \\ \text { GA } & \text { gestational age } \\ \text { APGAR score } & \text { appearance, pulse, grimace, activity, and respiration } \\ \text { RDS } & \text { respiratory distress syndrome } \\ \text { NEC } & \text { necrotizing enterocolitis } \\ \text { IVH } & \text { intraventricular hemorrhage } \\ \text { MRI } & \text { magnetic resonance imaging } \\ \text { BPD } & \text { bronchopulmonary dysplasia } \\ \text { CPAP } & \text { continuous positive airway pressure } \\ \text { SD } & \text { standard deviation }\end{array}$

\section{References}

1. Chawanpaiboon, S.; Vogel, J.P.; Moller, A.-B.; Lumbiganon, P.; Petzold, M.; Hogan, D.; Landoulsi, S.; Jampathong, N.; Kongwattanakul, K.; Laopaiboon, M.; et al. Global, regional, and national estimates of levels of preterm birth in 2014: A systematic review and modelling analysis. Lancet Glob. Health 2019, 7, e37-e46. [CrossRef]

2. Blencowe, H.; Cousens, S.; Chou, D.; Oestergaard, M.; Say, L.; Moller, A.B.; Kinney, M.; Lawn, J.; Born Too Soon Preterm Birth Action Group. Born too soon: The global epidemiology of 15 million preterm births. Reprod. Health 2013, 10. [CrossRef] [PubMed] 
3. Callaghan, W.M.; MacDorman, M.F.; Rasmussen, S.A.; Qin, C.; Lackritz, E.M. The Contribution of Preterm Birth to Infant Mortality Rates in the United States. Pediatrics 2006, 118, 1566-1573. [CrossRef]

4. Bhutta, Z.A.; Chopra, M.; Axelson, H.; Berman, P.; Boerma, T.; Bryce, J.; Bustreo, F.; Cavagnero, E.; Cometto, G.; Daelmans, B.; et al. Countdown to 2015 decade report (2000-10): Taking stock of maternal, newborn, and child survival. Lancet 2010, 375, $2032-2044$. [CrossRef]

5. Simonsen, K.A.; Anderson-Berry, A.L.; Delair, S.F.; Dele Davies, H. Early-Onset Neonatal Sepsis. Clin. Microbiol. Rev. 2014, 27, 21-47. [CrossRef] [PubMed]

6. Ku, L.C.; Boggess, K.A.; Cohen-Wolkowiez, M. Bacterial Meningitis in Infants. Clin. Perinatol. 2015, 42, 29-45. [CrossRef] [PubMed]

7. Null, N. Characterisation and antimicrobial resistance of sepsis pathogens in neonates born in tertiary care centres in Delhi, India: A cohort study. Lancet Glob. Health 2016, 4, e752-e760. [CrossRef]

8. Glikman, D.; Curiel, N.; Glatman-Freedman, A.; Megged, O.; Youngster, I.; Marom, R.; Lavie, K.; Smolkin, T.; Troitzky, M.; Stein, M.; et al. Nationwide epidemiology of early-onset sepsis in Israel 2010-2015, time to re-evaluate empiric treatment. Acta Paediatr. 2019, 108, 2192-2198. [CrossRef]

9. Klinger, G.; Levy, I.; Sirota, L.; Boyko, V.; Reichman, B.; Lerner-Geva, L. Epidemiology and risk factors for early onset sepsis among very-low-birthweight infants. Am. J. Obstet. Gynecol. 2009, 201, 38.e1-38.e6. [CrossRef]

10. Stoll, B.J.; Hansen, N.I.; Sánchez, P.J.; Faix, R.G.; Poindexter, B.B.; Van Meurs, K.P.; Bizzarro, M.J.; Goldberg, R.N.; Frantz, I.D.; Hale, E.C.; et al. Early Onset Neonatal Sepsis: The Burden of Group B Streptococcal and E. coli Disease Continues. Pediatrics 2011, 127, 817-826. [CrossRef]

11. Zhu, M.; Jin, Y.; Duan, Y.; He, M.; Lin, Z.; Lin, J. Multi-Drug Resistant Escherichia coli Causing Early-Onset Neonatal Sepsis-A Single Center Experience from China. Infect. Drug Resist. 2019, 12, 3695-3702. [CrossRef] [PubMed]

12. Marando, R.; Seni, J.; Mirambo, M.M.; Falgenhauer, L.; Moremi, N.; Mushi, M.F.; Kayange, N.; Manyama, F.; Imirzalioglu, C.; Chakraborty, T.; et al. Predictors of the extended-spectrum-beta lactamases producing Enterobacteriaceae neonatal sepsis at a tertiary hospital, Tanzania. Int. J. Med. Microbiol. 2018, 308, 803-811.

13. Hillier, S.L.; Krohn, M.A.; Kiviat, N.B.; Watts, D.H.; Eschenbach, D.A. Microbiologic causes and neonatal outcomes associated with chorioamnion infection. Am. J. Obstet. Gynecol. 1991, 165, 955-961. [CrossRef]

14. Jalilian, N.; Kooshkiforooshani, M.; Ahmadi, S.; Nankali, A. Colonisation with extended-spectrum $\beta$-lactamase-producing Enterobacteriaceae in pregnant/post-partum women: Systematic review and meta-analysis. J. Glob. Antimicrob. Resist. 2019, 19, 338-347. [CrossRef] [PubMed]

15. Villar, H.E.; Aubert, V.; Baserni, M.N.; Jugo, M.B. Maternal carriage of extended-spectrum beta-lactamase-producing Escherichia coliisolates in Argentina. J. Chemother. 2013, 25, 324-327. [CrossRef]

16. Bulabula, A.N.; Dramowski, A.; Mehtar, S. Maternal colonization or infection with extended-spectrum beta-lactamase-producing Enterobacteriaceae in Africa: A systematic review and meta-analysis. Int. J. Infect. Dis. 2017, 64, 58-66. [CrossRef]

17. Rettedal, S.; Lohr, I.H.; Bernhoff, E.; Natas, O.; Sundsfjord, A.; Oymar, K. Extended-spectrum $\beta$-lactamase-producing Enterobacteriaceae among pregnant women in Norway: Prevalence and maternal-neonatal transmission. J. Perinatol. 2015, 35, 907-912. [CrossRef] [PubMed]

18. Herindrainy, P.; Rabenandrasana, M.A.N.; Andrianirina, Z.Z.; Rakotoarimanana, F.M.J.; Padget, M.; De Lauzanne, A.; Ndir, A.; Kermorvant-Duchemin, E.; Garin, B.; Piola, P.; et al. Acquisition of extended spectrum beta-lactamase-producing enterobacteriaceae in neonates: A community based cohort in Madagascar. PLoS ONE 2018, 13, e0193325. [CrossRef]

19. Romero, R.; Mazor, M.; Munoz, H.; Gomez, R.; Galasso, M.; Sherer, D.M. The Preterm Labor Syndrome. Ann. N. Y. Acad. Sci. 1994, 734, 414-429. [CrossRef] [PubMed]

20. Romero, R.; Espinoza, J.; Gonçalves, L.F.; Kusanovic, J.P.; Friel, L.A.; Nien, J.K. Inflammation in preterm and term labour and delivery. Semin. Fetal Neonatal Med. 2006, 11, 317-326. [CrossRef] [PubMed]

21. Nadeau, H.C.; Subramaniam, A.; Andrews, W.W. Infection and preterm birth. Semin. Fetal Neonatal Med. 2016, 21, 100-105. [CrossRef]

22. Bastek, J.A.; Gómez, L.M.; Elovitz, M.A. The Role of Inflammation and Infection in Preterm Birth. Clin. Perinatol. 2011, 38, 385-406. [CrossRef] [PubMed]

23. Kim, Y.A.; Lee, K.; Chung, J.E. Risk factors and molecular features of sequence type (ST) 131 extended-Spectrum- $\beta$-lactamaseproducing Escherichia coli in community-onset female genital tract infections. BMC Infect. Dis. 2018, 18, 250. [CrossRef]

24. Hornsey, M.; Phee, L.; Woodford, N.; Turton, J.; Meunier, D.; Thomas, C.; Wareham, D.W. Evaluation of three selective chromogenic media, CHROMagar ESBL, CHROMagar CTX-M and CHROMagar KPC, for the detection of Klebsiella pneumoniae producing OXA-48 carbapenemase. J. Clin. Pathol. 2013, 66, 348-350. [CrossRef] [PubMed]

25. Danino, D.; Melamed, R.; Sterer, B.; Porat, N.; Hazan, G.; Gushanski, A.; Shany, E.; Greenberg, D.; Borer, A. Mother-to-child transmission of extended-spectrum-beta-lactamase-producing Enterobacteriaceae. J. Hosp. Infect. 2018, 100, 40-46. [CrossRef]

26. Platteel, T.; Hall, M.L.-V.; Stuart, J.C.; Thijsen, S.; Mascini, E.; Van Hees, B.; Scharringa, J.; Fluit, A.; Bonten, M. Predicting carriage with extended-spectrum beta-lactamase-producing bacteria at hospital admission: A cross-sectional study. Clin. Microbiol. Infect. 2015, 21, 141-146. [CrossRef] [PubMed]

27. Vornhagen, J.; Quach, P.; Boldenow, E.; Merillat, S.; Whidbey, C.; Ngo, L.Y.; Waldorf, K.M.A.; Rajagopal, L. Bacterial Hyaluronidase Promotes Ascending GBS Infection and Preterm Birth. mBio 2016, 7, e00781-16. [CrossRef] 
28. Denkel, L.A.; Gastmeier, P.; Piening, B. To screen or not to screen mothers of preterm infants for extended-spectrum betalactamase-producing Enterobacteriaceae (ESBL-E). J. Perinatol. 2015, 35, 893-894. [CrossRef]

29. Peretz, A.; Skuratovsky, A.; Khabra, E.; Adler, A.; Pastukh, N.; Barak, S.; Perlitz, Y.; Ben-Ami, M.; Kushnir, A. Peripartum maternal transmission of extended-spectrum $\beta$-lactamase organism to newborn infants. Diagn. Microbiol. Infect. Dis. 2017, 87, 168-171. [CrossRef]

30. Rettedal, S.; Löhr, I.H.; Natås, O.; Sundsfjord, A.; Øymar, K. Risk factors for acquisition of CTX-M-15 extended-spectrum betalactamase-producing Klebsiella pneumoniae during an outbreak in a neonatal intensive care unit in Norway. Scand. J. Infect. Dis. 2013, 45, 54-58. [CrossRef] [PubMed]

31. Boo, N.Y.; Ng, S.F.; Lim, V.K.E. A case-control study of risk factors associated with rectal colonization of extended-spectrum beta-lactamase producing Klebsiella sp. in newborn infants. J. Hosp. Infect. 2005, 61, 68-74. [CrossRef] [PubMed]

32. American Academy of Pediatrics. Prevention of Group B Streptococcal Early-Onset Disease in Newborns. Obstet. Gynecol. 2020, 135, e51-e72. [CrossRef] [PubMed]

33. Regan, J.A.; Klebanoff, M.A.; Nugent, R.P. The epidemiology of group B streptococcal colonization in pregnancy. Vaginal Infections and Prematurity Study Group. Obstet. Gynecol. 1991, 77, 604-610. [PubMed]

34. Campbell, J. Group B streptococcal colonization and serotype-specific immunity in pregnant women at delivery. Obstet. Gynecol. 2000, 96, 498-503. [CrossRef] [PubMed]

35. Singleton, A.; Cluck, D. The Pharmacist's Role in Treating Extended-Spectrum Beta-Lactamase Infections. Available online: https: //www.uspharmacist.com/article/the-pharmacists-role-in-treating-extendedspectrum-betalactamase-infections (accessed on 29 January 2021). 